\title{
Recent Underlying Event Studies at CDF
}

\section{Deepak Kar*}

Institut fur Kern- und Teilchenphysik, TU Dresden

E-mail: deepak.karecern.ch

\section{Rick Field}

Department of Physics, University of Florida

E-mail: rfieldephys.ufl.edu

CDF Run II data for the underlying event associated with Drell-Yan lepton pair production are examined as a function of the lepton-pair transverse momentum. The data are compared with a previous analysis on the behavior of the underlying event in high transverse momentum jet production and also with several other QCD Monte-Carlo models. The goal is to provide data that can be used to tune and improve the QCD Monte-Carlo models of the underlying event, which is especially important now in view of the LHC startup. Also, predictions of underlying event activity at LHC energies are shown.

XXth Hadron Collider Physics Symposium

November 16 - 20, 2009

Evian, France

${ }^{*}$ Speaker. 

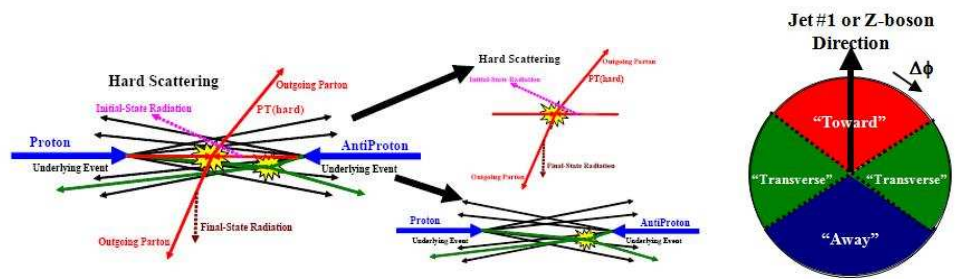

Figure 1: A typical 2-2 hard scattering process and dividing the central region

\section{Introduction: the Underlying Event}

In order to find 'new' physics at a hadron-hadron collider it is essential to have Monte-Carlo models that simulate accurately the 'ordinary' QCD hard-scattering events. To do this one must not only have a good model of the hard scattering part of the process, but also of the underlying event.

A typical 2-to-2 hard scattering event is a proton-antiproton collision at the hadron colliders as shown in the Figure 1(a), all happening inside the radius of a proton. In addition to the two hard scattered outgoing partons, which fragment into jets - there is initial and final state radiation (caused by bremsstrahlung and gluon emission), multiple parton interaction (additional 2-to-2 scattering within the same event), 'beam beam remnants' (particles that come from the breakup of the proton and antiproton, from the partons not participating in the primary hard scatter). We define the 'underlying event' [1] as everything except the hard scattered components, which includes the 'beam-beam remnants' (or the BBR) plus the multiple parton interaction (or the MPI). However, it is not possible on an event-by-event basis to be certain which particles came from the underlying event and, which particles originated from the hard scattering. The 'underlying event' (i.e. BBR plus MPI) is an unavoidable background to most collider observables. For example, at the Tevatron both the inclusive jet cross section and the b-jet cross section, as well as isolation cuts and the measurement of missing energy depend sensitively on the underlying event. A good understanding of it will lead to more precise measurements at the Tevatron and the LHC.

For Drell-Yan lepton pair production, we have the outgoing lepton anti-lepton pair in the final state and there would be no colored final state radiation. Hence it provides a very clean way to study the underlying event.

\section{The Underlying Event as a Function of Lepton Pair $p_{T}$}

We looked at the charged particles in the range $p_{T}>0.5 \mathrm{GeV} / \mathrm{c}$ and $|\eta|<1$, at the region of Z-boson, defined as $70 \mathrm{GeV} / c^{2}<M_{l l}<110 \mathrm{GeV} / \mathrm{c}^{2}$, in the 'toward', 'away' and 'transverse' regions, as defined in Fig. 1(b). The underlying event observables are found to be reasonably flat with the increasing lepton pair transverse momentum in the transverse and toward regions, but goes up in the away region to balance the lepton pairs. In Fig. 2(a) and Fig. 2(b), we looked at the two observables corresponding to the underlying event, the number of charged particle density and the charged transverse momentum sum density in the transverse region, compared with PYTHIA tunes A and AW [3], HERWIG [4] without MPI and a previous CDF analysis on leading jet underlying event results. We mostly observed very good agreements with PYTHIA tune AW Monte Carlo 

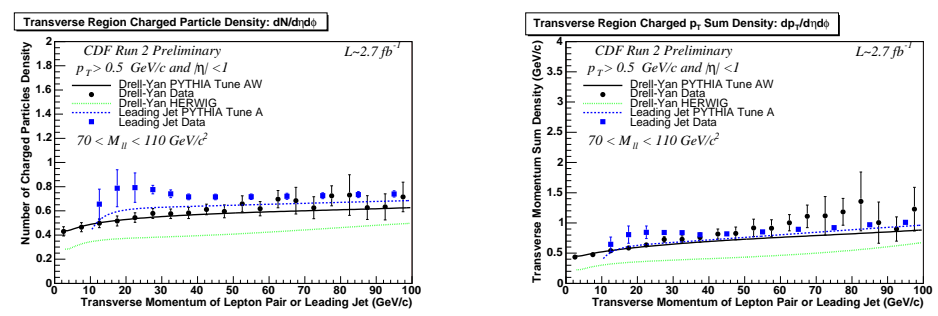

Figure 2: Drell-Yan underlying event plots, charged particle multiplicity on the left and the charged $p_{T}$ sum on the right

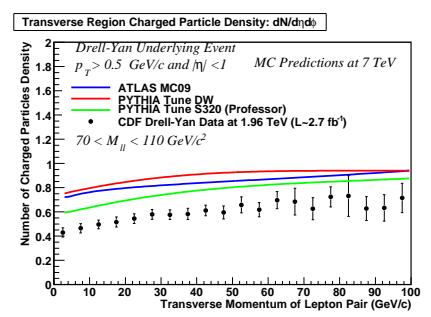

Figure 3: LHC Extrapolations at $7 \mathrm{TeV}$ from different Monte-Carlo tunes

predictions (HERWIG produces much less activity), although the agreement between theory and data is not perfect. We also compared them with leading jet underlying event results and observed reasonably close agreement - which may indicate the universality of underlying event modeling.

\section{Summary and Conclusions}

We are making good progress in understanding and modeling the softer physics. CDF tunes $\mathrm{A}$ and AW describe the data very well, although we still do not yet have a perfect fit to all the features of the CDF underlying event and min-bias data. CDF underlying event data has been used extensively for Monte-Carlo tuning by ATLAS, CMS and automated tuning tools like PROFESSOR [5]. Underlying event measurement plan at LHC benefits from the experience at CDF and LHC predictions are based on the extrapolations from mostly Tevatron data as in Fig. 3. Underlying event would be much more active at LHC and one of the earliest measurements, since we need to 'rediscover' the standard model in order to find any new physics. It is critical to have sensible underlying event models containing our best physical knowledge and intuition, tuned to all relevant available data.

\section{References}

[1] D. Acosta et al., Phys. Rev. D 70072002 (2004).

[2] T. Sjostrand et al., Computer Physics Commun. 135238 (2001).

[3] Rick. Field et al., [hep-ph/0510198].

[4] G. Corcella et al., JHEP 110 (2001).

[5] A. Buckley et al., Eur. Phys. J. C 65331 (2010). 